\title{
Exosomes in breast milk: a genetic trojan horse from mother to child
}

\author{
Dimitrios Vlachakis ${ }^{1,2,3 凶}$, Aspasia Efthimiadou ${ }^{4}$, Flora Bacopoulou ${ }^{5}$, Elias Eliopoulos ${ }^{1}$, George P. Chrousos ${ }^{5}$ \\ ${ }^{1}$ Laboratory of Genetics, Department of Biotechnology, School of Applied Biology and Biotechnology, Agricultural University of \\ Athens, Athens, Greece \\ ${ }^{2}$ Laboratory of Molecular Endocrinology, Center of Clinical, Experimental Surgery and Translational Research, Biomedical Research \\ Foundation of the Academy of Athens, Athens, Greece \\ ${ }^{3}$ School of Informatics, Faculty of Natural \& Mathematical Sciences, King's College London, London, United Kingdom \\ ${ }^{4}$ Hellenic Agricultural Organization-Demeter, Institute of Soil and Water Resources, Department of Soil Science of Athens, Lycovrisi, \\ Greece \\ ${ }^{5}$ University Research Institute of Maternal and Child Health \& Precision Medicine, and UNESCO Chair on Adolescent Health Care, \\ National and Kapodistrian University of Athens, Aghia Sophia Children's Hospital, Athens, Greece \\ Competing interests: DV none; AE none; FB none; EE none; GPC none
}

Breast milk is the ideal food for premature and mature babies and has undoubtedly immediate and ultimate benefits (Haschke et al., 2016). Among other things, it protects against infections, reduces the risk of necrotising enterocolitis and retinopathy of premature babies, improves neurodevelopmental outcome, and reduces the risk of obesity and metabolic syndrome later in life (Oddy 2002). In the present study, breast milk is being studied with all the available omics technologies available. More specifically, functional genomics, comparative genomics, transcriptomics, sequencing, proteomics, and metabolomics are applied. The above results and this multidimensional information are coordinated under the framework of a holistic approach of systems biology and bioinformatic analysis. Important lncRNAs and protein molecules are being validated as candidate biomarkers in exosomes of a larger group of breast milk and blood/serum samples. Validated ncRNAs / proteins are being analysed in exudates of breast milk, bovine, goat, and sheep milk to explore new ways to understand the genetics underlying breast milk. The expression of ncRNAs, unlike mRNAs, is a direct indicator of their functional presence. The information generated in this study is analysed by data mining and data combining techniques and algorithms. The benefits of breast milk are attributed to its various components, including nutrients, hormones, growth factors, immune cells, antibodies, cytokines, antimicrobial peptides, and extracellular vesicles (O’Reilly et al., 2021). Breast milk molecular fingerprinting will pave the way to shed light on the underlying genetics and epigenetics that a mother offers to her child.

\section{Acknowledgements}

This research has been co-financed by the European Regional Development Fund of the European Union and Greek national funds through the Operational Program Competitiveness, Entrepreneurship and Innovation, under the call RESEARCH - CREATE - INNOVATE (project code:T2EDK-02222).

\section{References}

1. Haschke F, Haiden N and Thakkar SK (2016) Nutritive and Bioactive Proteins in Breastmilk. Ann Nutr Metab 69 Suppl 2, 17-26. http://dx.doi.org/10.1159/000452820

2. O'Reilly D, Dorodnykh D, Avdeenko NV, Nekliudov NA, Garssen J et al. (2021) Perspective: The Role of Human Breast-Milk Extracellular Vesicles in Child Health and Disease. Adv Nutr 12(1), 59-70. http://dx.doi.org/10.1093/advances/nmaa094

3. Oddy WH (2002) The impact of breastmilk on infant and child health. Breastfeed Rev 10(3), 5-18. 